\title{
Biological and behavioral predictors of compensatory energy intake after exercise
}

\author{
Christoph Höchsmann \\ Technical University of Munich https://orcid.org/0000-0003-2007-3007 \\ Safiya E Beckford \\ University of Nebraska-Lincoln https://orcid.org/0000-0003-1886-9348 \\ Jeffrey A French \\ University of Nebraska Omaha https://orcid.org/0000-0001-5304-1592 \\ Julie B Boron \\ University of Nebraska Omaha https://orcid.org/0000-0003-1121-8120 \\ Jeffrey R Stevens \\ University of Nebraska-Lincoln https://orcid.org/0000-0003-2375-1360 \\ Karsten Koehler ( $\nabla$ karsten.koehler@tum.de) \\ Technical University of Munich https://orcid.org/0000-0002-9618-2069
}

\section{Research Article}

Keywords: compensatory eating, test meal, aerobic exercise, habitual exercise, appetite hormones

Posted Date: March 7th, 2022

DOI: https://doi.org/10.21203/rs.3.rs-1414640/v1

License: (c) (i) This work is licensed under a Creative Commons Attribution 4.0 International License. Read Full License 


\section{Abstract}

Energy intake in the post-exercise state is highly variable and compensatory eating - i.e., (over-) compensation of the expended energy via increased postexercise energy intake - occurs in some individuals but not others. We aimed to identify predictors of post-exercise energy intake and compensation. In a randomized crossover design, 57 healthy participants $(21.7$ [SD $=2.5]$ years; $23.7[\mathrm{SD}=2.3] \mathrm{kg} / \mathrm{m}^{2}, 75 \%$ White, 54\% female) completed two laboratory-based test-meals following (1) 45-min exercise and (2) 45-min rest (control). We assessed associations between biological (sex, body composition, appetite hormones) and behavioral (habitual exercise via prospective exercise log, appetitive traits) characteristics at baseline and total energy intake, compensatory energy intake (intake - exercise expenditure), and the difference between post-exercise and post-rest intake. We found a differential impact of biological and behavioral characteristics on total post-exercise energy intake in men and women. In men, only fasting (baseline) concentrations of appetite-regulating hormones (peptide $Y Y[P Y Y, \beta=0.88, P<0.001]$ and adiponectin $[\beta=0.66, P=0.005]$ predicted total post-exercise energy intake, while in women, only habitual exercise $(\beta=-0.44, P=0.017)$ predicted total post-exercise energy intake. Predictors of compensatory intake (intake - exercise expenditure) were almost identical to those of total intake. The difference in energy intake between exercise and rest was associated with $\mathrm{VO}_{2 \text { peak }}(\beta=-0.45, P=0.020)$, fasting PYY $(\beta=0.53, P=0.036)$, and fasting adiponectin $(\beta=0.57, P=0.021)$ in men but not women (all $\left.P>0.51\right)$. Our results show that biological and behavioral characteristics differentially affect total and compensatory post-exercise energy intake in men and women. This may help identify individuals who are more likely to compensate for the energy expended in exercise. Targeted countermeasures to prevent compensatory energy intake after exercise should take the demonstrated sex differences into account.

\section{Introduction}

Regular physical activity (PA) and exercise are recommended as methods of weight control; however, there is substantial variability in post-exercise energy intake. While some individuals show reduced energy intake post-exercise, allowing for an exercise-induced energy deficit, others show a compensatory increase in energy intake which negates the potential for exercise to promote negative energy balance and subsequent weight loss. ${ }^{1,2}$ Consequently, the effectiveness of exercise as a method of weight management is highly variable. ${ }^{3,4}$ Biological and behavioral participant characteristics may play a role in the energy intake response to exercise. Therefore, the objective of the present exploratory analyses was to assess predictors of ad libitum energy intake during a laboratory-based test-meal in healthy participants following a single 45-minute aerobic exercise bout. Specifically, we aimed to assess predictors of (1) total test-meal energy intake ( $\mathrm{kcal}$ ) and (2) compensatory energy intake ( $\mathrm{kcal}$ ), i.e., test-meal energy intake relative to the energy expended during the exercise bout. Additionally, as control, we aimed to identify predictors of the difference in energy intake between the post-exercise test-meal and an ad libitum test-meal after 45 minutes of rest.

We investigated the contributions of sex, anthropometrics, behavioral characteristics, and physiological/endocrine factors at baseline to compensatory ad libitum post-exercise energy intake. As energy balance is better regulated in individuals with an active lifestyle compared to those with a sedentary lifestyle,

${ }^{5-7}$ we hypothesized that greater levels of habitual activity and exercise would be associated with lower compensatory post-exercise energy intake. Further, appetitive traits such as cognitive restraint, uncontrolled eating, and emotional eating are associated with unintentional overeating in the presence of food $^{8,9}$ and we aimed to explore whether these general appetitive traits would also predict post-exercise energy intake. Finally, studies in lean individuals and those with overweight/obesity have shown that there is considerable variability in the appetite-regulating hormone responses to exercise, ${ }^{10-12}$ and we aimed to assess whether fasting concentrations of appetite-regulating hormones at baseline would be predictive of post-exercise energy intake.

Identification of such biological and behavioral baseline predictors would allow identifying individuals who are more likely to show exercise-induced energy compensation before these individuals engage in exercise and thereby pave the way for targeted countermeasures (e.g., cognitive-behavioral strategies, preparation of post-exercise meal ahead of time, or consumption of small meal before exercise) ahead of time.

We specifically sought to analyze the impact of fasting baseline concentrations of appetite-regulating hormones on post-exercise energy intake, as this would allow prediction of compensatory eating at the earliest time possible to maximize the weight loss potential of exercise.

\section{Materials And Methods}

\subsection{Study design and participants}

Following two preliminary assessment visits, this randomized crossover study involved two study conditions in random order that were completed on two separate days: (1) one 45-minute exercise bout and (2) one rest period of identical duration. Volunteers for this study were recruited from the University of Nebraska and its surrounding communities via fliers and word-of-mouth. Participants were eligible if they were 19-29 years old, had a body mass index (BMI) of $18.5-29.9 \mathrm{~kg} / \mathrm{m}^{2}$, were weight stable ( $\leq 2.5 \mathrm{~kg}$ weight change during the past six months), and exercised regularly ( $\geq 1$ bout/week). The complete list of inclusion and exclusion criteria has been published previously. ${ }^{13}$ All study procedures were approved by the University of Nebraska-Lincoln's Institutional Review Board (project number 17239) and written informed consent was obtained from all participants before participation in the study.

\subsection{Measures}

Anthropometric data, PA behavior, cardiorespiratory fitness, and appetitive traits were assessed during the preliminary assessment visits that occurred before participation in the study conditions, as published in detail previously. ${ }^{13}$ Briefly:

\subsubsection{Anthropometric data}


Weight and height were measured using a digital scale and stadiometer (Seca, Hamburg, Germany). Total body fat (\%) and fat-free mass (FFM, kg) were estimated via a 7-site skinfold assessment.

\subsubsection{Physical activity behavior}

PA behavior, and specifically moderate-to-vigorous PA (MVPA, min/week), was assessed over seven days using accelerometry (hip-worn GT3X+, Actigraph, Pensacola, FL). Participants were instructed to wear the GT3X + continuously throughout the 7-day monitoring period and to only remove it for swimming or taking a shower. Additionally, as it has been reported before that Actigraph devices are inaccurate at recording activities such as strength training and cycling, ${ }^{14-16}$ we instructed participants to prospectively record their habitual exercise ( $\mathrm{min} /$ week and days/week) over the same period using an exercise $\log$.

\subsubsection{Cardiorespiratory fitness}

Peak oxygen uptake $\left(\mathrm{VO}_{2 \text { peak }}\right)$ was measured using an incremental all-out exercise test on a bicycle ergometer (LC6, Monark, Vansbro, Sweden). Participants began cycling at a resistance of $60 \mathrm{~W}$ for 3 minutes, and the work rate was increased by $35 \mathrm{~W}$ every 3 minutes until exhaustion. ${ }^{17}$ Maximal exhaustion was accepted when at least two of the following were met: (1) Heart rate of $\geq 90 \%$ of age-predicted maximal heart rate, (2) a respiratory exchange ratio $\geq 1.1$, (3) rating of perceived exertion $\geq 19,{ }^{18}(4)$ a plateau in oxygen uptake despite the increasing workload. Throughout the test, respiratory gas parameters were analyzed breath by breath (Quark CPET, COSMED, Rome, Italy) and heart rate was monitored through telemetry (Polar, Kempele, Finland).

\subsubsection{Appetitive traits}

Cognitive restraint, uncontrolled eating, and emotional eating were assessed with the revised 18-item Three-Factor Eating Questionnaire (TFEQ-R18v2). The TFEQ-R18v2 is a shortened version of the original well-validated 51-item TFEQ by Stunkard and Messick, ${ }^{19}$ which has demonstrated improved psychometric properties, minimized floor and ceiling effects in the emotional eating domain, and improved internal consistency in the cognitive restraint domain compared to the earlier shortened versions of the TFEQ (TFEQ-R18 and TFEQ-R21), with an overall robust factor structure and good reliability in two large North American samples. ${ }^{20,21}$

\subsubsection{Appetitive-regulating hormones}

Fasting plasma concentrations of glucagon-like peptide 1 (GLP-1), ghrelin, peptide YY (PYY), and adiponectin were measured at each study condition visit immediately after arrival to the laboratory before participants received a standardized breakfast and continued with the initial 30 -minute rest period. Wholeblood samples were collected into ethylenediaminetetraacetic acid (EDTA) tubes from participants in a seated position. A protease inhibitor (aprotinin; Sigma Aldrich, St. Louis, MO) was added to PYY and ghrelin samples. Immediately after collection, the EDTA tubes were placed on ice for 15 minutes and then centrifuged at $1800 \times \mathrm{g}$ for 10 minutes at $+4^{\circ} \mathrm{C}$. Subsequently, plasma fractions were aliquoted and stored at $-80^{\circ} \mathrm{C}$ until analysis. Enzyme-linked immunosorbent assays (ELISAs) were used to measure concentrations of PYY (Millipore Sigma, Burlington, MA) and GLP-1, ghrelin, and adiponectin (all Invitrogen ${ }^{\mathrm{TM}}$, Carlsbad, CA).

\subsection{Study conditions}

On the day of each study condition, participants arrived at the lab between 06:30 and 10:00, following an overnight fast and abstinence from alcohol for at least 24 hours. Participants further refrained from exercise and strenuous physical activity the day before and the morning of their visits, with compliance monitored via accelerometry (GT3X+, Actigraph, Pensacola, FL). During their first study condition visit, participants completed a 24-hour diet recall using an Automated Self-Administered 24-hour Dietary Assessment Tool (ASA24, National Cancer Institute, Bethesda, MD, USA). Participants received a copy of their recall after the visit and they were instructed to replicate the diet as closely as possible on the day before their second study condition visit. At each study condition visit, participants were provided with a small standardized breakfast (commercially available cereal bar [240 kcal] and 8 ounces of bottled water) upon arrival and instructed to rest for 30 minutes in a seated position.

\subsubsection{Exercise condition}

Following the initial 30-minute rest, participants exercised on a bicycle ergometer (LC6, Monark, Vansbro, Sweden) for 45 minutes at an intensity of $60 \%$ of their $\mathrm{VO}_{2 \text { peak. }}$. Heart rate and ratings of perceived exertion ${ }^{18}$ were monitored at regular intervals throughout the exercise bout. After completion of the exercise bout, participants rested for another 30 minutes before being offered the test meal.

\subsubsection{Rest condition}

For the resting condition, participants were instructed to sit quietly in a chair for 45 minutes, following the initial 30 -minute rest period. To ensure an overall identical timing and sequence of the two study condition visits, participants rested for an additional 30 minutes after the 45-minute rest condition before being offered the test meal. Throughout both visits, participants were allowed to listen to music or watch pre-approved TV programs that did not contain any images of or references to food.

\subsubsection{Ad libitum test-meal}

Thirty minutes after each study condition (exercise or rest), participants were offered an identical single-item ad libitum test meal. The test meal (frozen family-size cheese pizza, HyVee, West Des Moines, IA) was prepared by study staff, and participants were offered the entire pizza ( 3,200 kcal, above energy needs) at once. The test meal was consumed in a separate room and under supervision, and cell phone use was restricted. Participants were 
instructed to eat as much or as little of the test meal as they would like and to make sure to eat the pre-cut pizza slices evenly (i.e., not leave/discard the crust or take the cheese off, etc.). Pre- and post-meal weights (grams) were recorded, with the difference in weight representing food intake. Gram weights were converted to energy intake (kcal) using the pizza's nutrition label.

\subsection{Statistical Analyses}

The distribution of variables was verified by visual inspection of histograms and quantile-quantile plots of the residuals. Exclusion of outliers ( $\leq 2$ for all models) did not change the results meaningfully; therefore, the models including outliers are reported. Descriptive data are reported as mean and standard deviation (SD). We used simple linear regression models to estimate the effect of anthropometric characteristics (weight, BMI, FFM, percent body fat), physiological characteristics ( $\mathrm{VO}_{2 \text { peak, }}$, maximal power, and fasting concentrations of appetite-regulating hormones such as GLP-1, ghrelin, PYY, and adiponectin), and behavioral characteristics (habitual exercise behavior, MVPA, and appetitive traits) on energy intake during an ad libitum single-item test meal. Specifically, we used the following four variables as dependent variables in our models: (1) post-exercise energy intake (kcal), (2) compensatory energy intake, which was defined as post-exercise energy intake [kcal] - energy expenditure during exercise session [kcal], (3) energy intake following the rest condition ( $\mathrm{kcal})$, and (4) the difference in energy intake between the post-exercise and the post-rest test meal (post-exercise energy intake [kcal] - postrest energy intake $[\mathrm{kcal}])$. Because it has been demonstrated in several studies that FFM is a predictor of meal size and single meal food intake, ${ }^{22-24}$ we $^{2}$ included FFM as a covariate in sensitivity analyses; however, results did not differ meaningfully and we consequently report the results without FFM as a covariate. Further, results for appetitive traits (cognitive restraint, uncontrolled eating, and emotional eating) based on the TFEQ-R18 and TFEQ-R21 did not differ meaningfully from the results of the TFEQ-R18v2 presented herein. Because the TFEQ-R18v2 has been validated in North American samples and improved internal consistency has been reported, as described above, only these results are reported. We used SPSS Statistics for Windows, version 27 (IBM Corp., Armonk, NY) for our analyses, and results were considered significant at $P<0.05$.

\section{Results}

\subsection{Participant characteristics}

Sixty-five participants were enrolled in the study. Eight participants were excluded (intensity of exercise session not at $60 \% \mathrm{VO}_{2 p e a k}[\mathrm{n}=6]$, no exercise data $[n=2]$ ); hence 57 participants were included in our analyses. Baseline characteristics of all included participants (mean age 21.7 [SD = 2.5] years, mean BMI 23.7 [SD $=2.3$ ], 75\% White, $54 \%$ female) are presented in Table 1. Weight, percent body fat, $F F M, V_{2}$ peak, and maximal power differed by sex (all $P \leq 0.001$ ), all other characteristics did not differ between men and women (all $P \geq 0.26$ ). On average, participants expended 343 (SD $=85$ ) kcal during the 45 -minute exercise session and consumed $867(S D=411) \mathrm{kcal}$ during the post-exercise ad libitum test meal. Relative to the energy expended during the exercise sessions, participants consumed $526(\mathrm{SD}=406) \mathrm{kcal}$ during the post-exercise test meal (compensatory intake). After the rest condition, energy intake during the test meal was $821(\mathrm{SD}=383) \mathrm{kcal}$ with an average difference in intake between the two test meals of $46(\mathrm{SD}=303) \mathrm{kcal}(P=0.26)$. Exerciserelated energy expenditure and energy intake during the test meals by sex are presented in Table 1. 
Table 1

Participant characteristics.

\begin{tabular}{|c|c|c|c|c|c|c|}
\hline & \multicolumn{2}{|c|}{ All $(N=57)$} & \multicolumn{2}{|c|}{ Men $(n=26)$} & \multicolumn{2}{|c|}{ Women $(n=31)$} \\
\hline \multicolumn{7}{|l|}{ Race/Ethinicity, n (\%) } \\
\hline White & 43 & $(75.4)$ & 18 & $(69.3)$ & 25 & $(80.6)$ \\
\hline African American & 9 & $(15.8)$ & 6 & $(23.1)$ & 3 & $(9.7)$ \\
\hline Asian & 4 & (7.0) & 1 & (3.8) & 3 & $(9.7)$ \\
\hline \multirow[t]{2}{*}{ Other } & 1 & $(1.8)$ & 1 & (3.8) & 0 & $(0.0)$ \\
\hline & Mean & (SD) & Mean & (SD) & Mean & (SD) \\
\hline Age, years & 21.7 & $(2.5)$ & 21.4 & $(2.4)$ & 21.9 & $(2.6)$ \\
\hline Weight, kg & 68.7 & $(10.2)$ & 73.6 & (11.3) & 64.6 & $(7.0)$ \\
\hline $\mathrm{BMI}, \mathrm{kg} / \mathrm{m}^{2}$ & 23.7 & $(2.3)$ & 23.8 & $(2.7)$ & 23.5 & $(2.1)$ \\
\hline Fat-free mass, kg & 59.6 & $(9.0)$ & 66.0 & $(8.9)$ & 54.2 & $(4.6)$ \\
\hline Total body fat, \% & 13.2 & $(6.0)$ & 9.9 & $(5.4)$ & 15.9 & $(5.1)$ \\
\hline \multicolumn{7}{|c|}{ Physical activity behavior and cardiorespiratory fitness } \\
\hline Total habitual exercise, min/week ${ }^{a}$ & 245.9 & $(181.2)$ & 236.0 & $(137.7)$ & 254.4 & $(213.9)$ \\
\hline Habitual exercise days, days/week & 3.4 & $(1.9)$ & 3.7 & $(1.9)$ & 3.3 & $(2.0)$ \\
\hline MVPA, min/week & 332.4 & $(145.7)$ & 350.4 & $(159.9)$ & 317.3 & $(133.4)$ \\
\hline Relative $\mathrm{VO}_{2 \text { peak }}, \mathrm{mL} / \mathrm{kg} / \mathrm{min}$ & 37.4 & $(6.2)$ & 40.6 & $(5.8)$ & 34.7 & $(5.2)$ \\
\hline Absolute $\mathrm{VO}_{2 \text { peak }}, \mathrm{L} / \mathrm{min}$ & 2.6 & $(0.6)$ & 2.3 & $(0.6)$ & 2.2 & $(0.3)$ \\
\hline Maximal power, $\mathrm{W}^{\mathrm{b}}$ & 220.6 & $(48.5)$ & 248.5 & $(47.6)$ & 196.5 & $(34.8)$ \\
\hline \multicolumn{7}{|l|}{ Appetitive traits } \\
\hline Cognitive Restraint via TFEQ-R18v2 & 5.9 & $(2.2)$ & 5.7 & $(2.6)$ & 6.0 & $(1.8)$ \\
\hline Uncontrolled Eating via TFEQ-R18v2 & 17.3 & $(4.7)$ & 17.6 & $(4.9)$ & 17.0 & $(4.5)$ \\
\hline Emotional Eating via TFEQ-R18v2 & 9.8 & (3.3) & 9.2 & $(2.7)$ & 10.2 & $(3.7)$ \\
\hline \multicolumn{7}{|l|}{ Appetite-regulating hormones ${ }^{c}$} \\
\hline GLP-1, pg/mL d & 10.9 & $(5.2)$ & 10.0 & $(4.4)$ & 11.4 & $(5.8)$ \\
\hline$P Y Y, p g / m L d$ & 110.8 & $(47.0)$ & 110.4 & $(46.5)$ & 111.1 & $(48.4)$ \\
\hline Ghrelin, pg/mL e & 865.2 & $(393.0)$ & 807.3 & $(435.0)$ & 906.5 & $(365.3)$ \\
\hline Adiponectin, $\mathrm{ng} / \mathrm{mL}^{f}$ & 11.7 & $(7.1)$ & 8.9 & $(6.4)$ & 13.8 & $(6.9)$ \\
\hline \multicolumn{7}{|l|}{ Exercise session } \\
\hline Energy expenditure, kcal & 343 & (85) & 400 & (85) & 296 & $(46)$ \\
\hline \multicolumn{7}{|l|}{ Test meal } \\
\hline Energy intake (post exercise), kcal & 867 & $(411)$ & 1010 & $(478)$ & 748 & $(304)$ \\
\hline Energy Intake (post rest), kcal & 821 & $(383)$ & 999 & $(376)$ & 672 & $(326)$ \\
\hline Difference in energy intake, $\mathrm{kcal} g$ & 46 & $(303)$ & 11 & $(398)$ & 75 & $(193)$ \\
\hline Compensatory energy intake, $\mathrm{kcal}^{\mathrm{h}}$ & 526 & $(406)$ & 609 & $(482)$ & 452 & $(320)$ \\
\hline
\end{tabular}

Data are mean (standard deviation) unless stated otherwise. Weight, percent body fat, $F F M, \mathrm{VO}_{2 \text { peak, }}$, and maximal power differed by sex (all $\mathrm{P} \leq 0.001$ ), all other characteristics did not differ between men and women (all $\mathrm{P} \geq 0.26$ ).

a Data available for $54 / 57$ participants (25/26 men and 29/31 women).

${ }^{b}$ Data available for $56 / 57$ participants (26/26 men and 30/31 women). 
${ }^{\mathrm{c}}$ Hormone concentrations are reported as means between pre-exercise and pre-rest. Fasting concentrations before the two study conditions did not differ (all $P \geq 0.08$ ).

d Data available for $39 / 57$ participants (16/26 men and 23/31 women).

e Data available for $36 / 57$ participants (15/26 men and 21/31 women).

${ }^{f}$ Data available for $38 / 57$ participants (16/26 men and 22/31 women).

${ }^{g}$ Post-exercise ad libitum energy intake (kcal) - energy intake following the rest condition (kcal).

${ }^{\mathrm{h}}$ Energy intake during test meal (kcal) - energy expenditure during exercise session (kcal)

Abbreviations: BMI, body mass index; GLP-1, Glucagon-like Peptide 1; MVPA, moderate-to-vigorous physical activity; PYY, peptide YY; SD, standard deviation; TFEQ-R18v2, revised 18-item Three-Factor Eating Questionnaire.

\subsection{Predictors of total post-exercise energy intake}

Total post-exercise energy intake was inversely associated with habitual exercise behavior $(\beta=-0.29, P=0.032$; Table 2, Figure 1A) and positively associated with FFM ( $\beta=0.30, P=0.025$; Table 2$)$ and fasting concentrations of PYY $(\beta=0.39, P=0.015$; Table 2, Figure 1D). We also found a sex effect, as men consumed on average $261.9 \mathrm{kcal}$ more than women $(P=0.015)$. After stratifying by sex, PYY $(\beta=0.88, P<0.001)$ and additionally adiponectin ( $\beta=0.66$, $P=0.005$, Table 2, Figure 1G) were significant predictors of total post-exercise energy intake only in men, while habitual exercise $(\beta=-0.44, P=0.017)$ was a significant predictor of total post-exercise energy intake only in women. 
Table 2

Linear regression analysis for the association between anthropometrics, physiological and behavioral baseline characteristics and total energy intake during the post-exercise ad libitum test meal.

\begin{tabular}{|c|c|c|c|c|c|c|c|c|c|c|c|c|c|c|c|}
\hline & \multicolumn{5}{|c|}{ All participants } & \multicolumn{5}{|l|}{ Men } & \multicolumn{5}{|c|}{ Women } \\
\hline & \multicolumn{5}{|c|}{ Energy intake (kcal) } & \multicolumn{5}{|c|}{ Energy intake (kcal) } & \multicolumn{5}{|c|}{ Energy intake (kcal) } \\
\hline & $\mathrm{R}^{2}$ & B & SE & $\beta$ & $\mathrm{P}$ & $\mathrm{R}^{2}$ & B & SE & $\beta$ & $\mathrm{P}$ & $\mathrm{R}^{2}$ & B & SE & $\beta$ & $\mathrm{P}$ \\
\hline Sex ${ }^{a}$ & 0.103 & 261.9 & 104.5 & 0.32 & 0.015 & & & & & & & & & & \\
\hline Age, years & 0.000 & -2.3 & 22.3 & -0.01 & 0.920 & 0.002 & -8.2 & 40.0 & -0.04 & 0.840 & 0.008 & 10.7 & 22.0 & 0.090 & 0.632 \\
\hline Weight, kg & 0.052 & 9.2 & 5.3 & 0.23 & 0.088 & 0.022 & 6.2 & 8.5 & 0.15 & 0.471 & 0.000 & 0.2 & 8.1 & 0.00 & 0.979 \\
\hline $\mathrm{BMI}, \mathrm{kg} / \mathrm{m}^{2}$ & 0.009 & 16.8 & 23.5 & 0.10 & 0.477 & 0.000 & 2.9 & 36.4 & 0.02 & 0.937 & 0.034 & 27.0 & 26.9 & 0.18 & 0.323 \\
\hline $\begin{array}{l}\text { Fat-free } \\
\text { mass, kg }\end{array}$ & 0.088 & 13.5 & 5.9 & 0.30 & 0.025 & 0.059 & 13.1 & 10.7 & 0.24 & 0.233 & 0.031 & -11.6 & 12.0 & -0.18 & 0.340 \\
\hline $\begin{array}{l}\text { Total body } \\
\text { fat, \% }\end{array}$ & 0.018 & -9.4 & 9.2 & -0.14 & 0.315 & 0.016 & -11.2 & 18.1 & -0.13 & 0.542 & 0.060 & 14.7 & 10.8 & 0.25 & 0.183 \\
\hline \multicolumn{16}{|c|}{ Physical activity behavior and cardiorespiratory fitness } \\
\hline $\begin{array}{l}\text { Habitual } \\
\text { exercise, } \\
\text { min/week }\end{array}$ & 0.085 & -0.7 & 0.3 & -0.29 & 0.032 & 0.032 & -0.6 & 0.7 & -0.18 & 0.395 & 0.194 & -0.6 & 0.3 & -0.44 & 0.017 \\
\hline $\begin{array}{l}\text { Habitual } \\
\text { exercise } \\
\text { days/week }\end{array}$ & 0.024 & -33.0 & 28.1 & -0.16 & 0.246 & 0.013 & -28.9 & 50.6 & -0.12 & 0.572 & 0.103 & -49.3 & 27.0 & -0.32 & 0.078 \\
\hline $\begin{array}{l}\text { MVPA, } \\
\text { min/week }\end{array}$ & 0.012 & 0.3 & 0.4 & 0.11 & 0.418 & 0.019 & 0.4 & 0.6 & 0.14 & 0.498 & 0.000 & 0.0 & 0.4 & -0.02 & 0.924 \\
\hline $\begin{array}{l}\text { Relative } \\
\mathrm{VO}_{2 \text { peak, }} \\
\mathrm{mL} / \mathrm{kg} / \mathrm{min}\end{array}$ & 0.000 & 1.2 & 9.0 & 0.02 & 0.896 & 0.009 & -7.8 & 16.8 & -0.09 & 0.648 & 0.074 & -16.0 & 10.5 & -0.27 & 0.138 \\
\hline $\begin{array}{l}\text { Absolute } \\
\mathrm{VO}_{2 \text { peak' }} \\
\mathrm{L} / \mathrm{min}\end{array}$ & 0.021 & 97.5 & 90.2 & 0.14 & 0.284 & 0.000 & 9.4 & 158.1 & 0.01 & 0.953 & 0.083 & -270.1 & 166.2 & -0.29 & 0.115 \\
\hline $\begin{array}{l}\text { Maximal } \\
\text { power, W }\end{array}$ & 0.049 & 1.9 & 1.1 & 0.22 & 0.101 & 0.015 & 1.2 & 2.0 & 0.12 & 0.558 & 0.003 & -0.5 & 1.7 & -0.05 & 0.781 \\
\hline \multicolumn{16}{|l|}{$\begin{array}{l}\text { Appetitive } \\
\text { traits }\end{array}$} \\
\hline $\begin{array}{l}\text { Cognitive } \\
\text { Restraint }\end{array}$ & 0.013 & -21.4 & 25.3 & -0.11 & 0.401 & 0.058 & -44.1 & 36.3 & -0.24 & 0.237 & 0.040 & 34.5 & 31.5 & 0.20 & 0.282 \\
\hline $\begin{array}{l}\text { Uncontrolled } \\
\text { Eating }\end{array}$ & 0.010 & 8.9 & 11.9 & 0.10 & 0.455 & 0.004 & -6.2 & 19.7 & -0.06 & 0.757 & 0.091 & 20.6 & 12.1 & 0.30 & 0.098 \\
\hline $\begin{array}{l}\text { Emotional } \\
\text { Eating }\end{array}$ & 0.002 & 5.8 & 16.9 & 0.05 & 0.732 & 0.011 & -18.0 & 35.5 & -0.10 & 0.618 & 0.097 & 26.0 & 14.7 & 0.31 & 0.087 \\
\hline
\end{tabular}

\section{Appetite-regulating hormones}

\begin{tabular}{|c|c|c|c|c|c|c|c|c|c|c|c|c|c|c|c|}
\hline GLP-1, pg/mL & 0.000 & 0.7 & 12.8 & 0.09 & 0.957 & 0.108 & 38.4 & 29.5 & 0.33 & 0.214 & 0.036 & -8.4 & 9.5 & -0.19 & 0.388 \\
\hline PYY, pg/mL & 0.149 & 3.3 & 1.3 & 0.39 & 0.015 & 0.775 & 9.6 & 1.4 & 0.88 & $<0.001$ & 0.013 & -0.6 & 1.1 & -0.12 & 0.600 \\
\hline Ghrelin, pg/mL & 0.005 & 0.1 & 0.2 & 0.07 & 0.681 & 0.087 & 0.3 & 0.3 & 0.30 & 0.285 & 0.021 & -0.1 & 0.2 & -0.14 & 0.535 \\
\hline Adiponectin, $\mathrm{ng} / \mathrm{mL}$ & 0.043 & 12.0 & 9.5 & 0.21 & 0.213 & 0.438 & 52.9 & 16.0 & 0.66 & 0.005 & 0.010 & 3.8 & 8.4 & 0.10 & 0.659 \\
\hline
\end{tabular}

Bold font indicates statistical significance $(P<0.05)$. Dependent variable in all models: Total energy intake during the post-exercise ad libitum test meal (kcal).

${ }^{\text {a }}$ Female $=0$, male $=1$

Abbreviations: B, unstandardized regression coefficient; $\beta$, standardized regression coefficient; BMI, body mass index; GLP-1, Glucagon-like Peptide 1; MVPA, moderate-to-vigorous physical activity; PYY, peptide YY; SE, standard error.

\subsection{Predictors of compensatory energy intake}


Similar to total post-exercise energy intake, compensatory energy intake was inversely associated with habitual exercise behavior $(\beta=-0.31, P=0.024$;

Table 3, Figure 1B) and positively associated with fasting concentrations of PYY ( $\beta=0.37, P=0.021$; Table 3, Figure 1E). Similar to total post-exercise energy intake, PYY $(\beta=0.85, P<0.001)$ and additionally adiponectin $(\beta=0.69, P=0.003$; Table 3 , Figure $1 \mathrm{H})$ were significant predictors of compensatory energy intake only in men, while habitual exercise ( $\mathrm{min} /$ week: $\beta=-0.44, P=0.016$; days/week: $\beta=-0.39, P=0.032$ ) and additionally $\mathrm{VO}_{2 \text { peak }}($ relative: $\beta=-0,36, P=0.044$; absolute: $\beta=-0.42 ; P=0.020$; Table 3) were significant predictors of compensatory energy intake only in women.

Table 3

Linear regression analysis for the association between anthropometrics, physiological and behavioral baseline characteristics and compensatory postexercise ad libitum energy intake (energy intake [kcal] - energy expenditure [kcal]).

\begin{tabular}{|c|c|c|c|c|c|c|c|c|c|c|c|c|c|c|c|}
\hline & \multicolumn{5}{|c|}{ All participants } & \multicolumn{5}{|l|}{ Men } & \multicolumn{5}{|c|}{ Women } \\
\hline & \multicolumn{5}{|c|}{ Compensatory energy intake (kcal) } & \multicolumn{5}{|c|}{ Compensatory energy intake (kcal) } & \multicolumn{5}{|c|}{ Compensatory energy intake (kcal) } \\
\hline & $\mathbf{R}^{2}$ & B & SE & $\boldsymbol{\beta}$ & $\mathrm{P}$ & $\mathrm{R}^{2}$ & B & SE & $\beta$ & $\mathrm{P}$ & $\mathrm{R}^{2}$ & B & SE & $\beta$ & $\mathrm{P}$ \\
\hline Sex ${ }^{a}$ & 0.038 & 157.4 & 106.9 & 0.19 & 0.147 & & & & & & & & & & \\
\hline Age, years & 0.000 & -2.0 & 22.0 & -0.01 & 0.929 & 0.007 & -16.1 & 40.3 & -0.08 & 0.693 & 0.012 & 13.8 & 23.1 & 0.11 & 0.554 \\
\hline Weight, kg & 0.007 & 3.4 & 5.4 & 0.09 & 0.528 & 0.000 & 0.9 & 8.7 & 0.02 & 0.923 & 0.002 & -2.0 & 8.5 & -0.04 & 0.816 \\
\hline $\mathrm{BMI}, \mathrm{kg} / \mathrm{m}^{2}$ & 0.000 & 3.0 & 23.3 & 0.02 & 0.899 & 0.007 & -14.5 & 36.6 & -0.08 & 0.695 & 0.020 & 21.8 & 28.5 & 0.14 & 0.449 \\
\hline $\begin{array}{l}\text { Fat-free } \\
\text { mass, kg }\end{array}$ & 0.019 & 6.1 & 6.0 & 0.14 & 0.312 & 0.013 & 6.1 & 11.0 & 0.11 & 0.583 & 0.054 & -16.0 & 12.5 & -0.23 & 0.209 \\
\hline \multicolumn{16}{|l|}{$\begin{array}{l}\text { Total body } \\
\text { fat, \% }\end{array}$} \\
\hline \multicolumn{16}{|c|}{ Physical activity behavior and cardiorespiratory fitness } \\
\hline $\begin{array}{l}\text { Habitual } \\
\text { exercise, } \\
\text { min/week }\end{array}$ & 0.094 & -0.7 & 0.3 & -0.31 & 0.024 & 0.035 & -0.6 & 0.7 & -0.19 & 0.372 & 0.197 & -0.7 & 0.3 & -0.44 & 0.016 \\
\hline $\begin{array}{l}\text { Habitual } \\
\text { exercise } \\
\text { days/week }\end{array}$ & 0.054 & -48.7 & 27.4 & -0.23 & 0.081 & 0.028 & -41.9 & 50.6 & -0.17 & 0.416 & 0.149 & -62.4 & 27.6 & -0.39 & 0.032 \\
\hline $\begin{array}{l}\text { MVPA, } \\
\text { min/week }\end{array}$ & 0.012 & 0.3 & 0.4 & 0.11 & 0.419 & 0.027 & 0.5 & 0.6 & 0.16 & 0.421 & 0.001 & -0.1 & 0.4 & -0.02 & 0.900 \\
\hline $\begin{array}{l}\text { Relative } \\
\mathrm{VO}_{2 \text { peak, }} \\
\mathrm{mL} / \mathrm{kg} / \mathrm{min}\end{array}$ & 0.019 & -9.1 & 8.8 & -0.14 & 0.306 & 0.043 & -17.3 & 16.7 & -0.21 & 0.312 & 0.133 & -22.6 & 10.7 & -0.36 & 0.044 \\
\hline $\begin{array}{l}\text { Absolute } \\
\mathrm{VO}_{2 \text { peak }} \\
\mathrm{L} / \mathrm{min}\end{array}$ & 0.004 & -40.1 & 89.9 & -0.06 & 0.657 & 0.026 & -127.0 & 157.4 & -0.16 & 0.428 & 0.173 & -408.6 & 166.0 & -0.42 & 0.020 \\
\hline $\begin{array}{l}\text { Maximal } \\
\text { power, W }\end{array}$ & 0.001 & 0.3 & 1.1 & 0.04 & 0.781 & 0.001 & -0.4 & 2.1 & -0.04 & 0.853 & 0.027 & -1.5 & 1.7 & -0.16 & 0.386 \\
\hline \multicolumn{16}{|l|}{$\begin{array}{l}\text { Appetitive } \\
\text { traits }\end{array}$} \\
\hline $\begin{array}{l}\text { Cognitive } \\
\text { Restraint }\end{array}$ & 0.012 & -20.4 & 25.0 & -0.11 & 0.420 & 0.050 & -41.2 & 36.8 & -0.22 & 0.274 & 0.021 & 26.4 & 33.4 & 0.15 & 0.436 \\
\hline $\begin{array}{l}\text { Uncontrolled } \\
\text { Eating }\end{array}$ & 0.008 & 7.7 & 11.7 & 0.09 & 0.517 & 0.008 & -8.5 & 19.9 & -0.09 & 0.671 & 0.094 & 22.0 & 12.7 & 0.31 & 0.093 \\
\hline $\begin{array}{l}\text { Emotional } \\
\text { Eating }\end{array}$ & 0.004 & 7.4 & 16.7 & 0.06 & 0.660 & 0.016 & -22.6 & 35.7 & -0.13 & 0.532 & 0.095 & 27.0 & 15.5 & 0.31 & 0.092 \\
\hline
\end{tabular}




\begin{tabular}{|c|c|c|c|c|c|c|c|c|c|c|c|c|c|c|c|}
\hline \multicolumn{16}{|c|}{ Appetiteregulating hormones } \\
\hline GLP-1, pg/mL & 0.002 & 3.0 & 12.5 & 0.04 & 0.811 & 0.108 & 38.8 & 29.8 & 0.33 & 0.214 & 0.024 & -7.5 & 10.3 & -0.16 & 0.477 \\
\hline $\mathrm{PYY}, \mathrm{pg} / \mathrm{mL}$ & 0.136 & 3.1 & 1.3 & 0.37 & 0.021 & 0.724 & 9.4 & 1.6 & 0.85 & $<0.001$ & 0.022 & -0.8 & 1.2 & -0.15 & 0.501 \\
\hline Ghrelin, pg/mL & 0.018 & 0.1 & 0.2 & 0.14 & 0.431 & 0.090 & 0.4 & 0.3 & 0.30 & 0.278 & 0.001 & -0.0 & 0.2 & -0.03 & 0.911 \\
\hline Adiponectin, $\mathrm{ng} / \mathrm{mL}$ & 0.066 & 14.6 & 9.2 & 0.26 & 0.120 & 0.476 & 55.8 & 15.6 & 0.69 & 0.003 & 0.001 & 1.3 & 9.0 & 0.03 & 0.888 \\
\hline
\end{tabular}

Bold font indicates statistical significance $(P<0.05)$. Dependent variable in all models: Compensatory post-exercise ad libitum energy intake (energy intake during test meal [kcal] - energy expenditure during exercise session [kcal]).

a Female $=0$, male $=1$

Abbreviations: B, unstandardized regression coefficient; $\beta$, standardized regression coefficient; BMI, body mass index; GLP-1, Glucagon-like Peptide 1; MVPA, moderate-to-vigorous physical activity; PYY, peptide YY; SE, standard error.

\subsection{Predictors of post-rest energy intake}

Energy intake after the rest condition was positively associated with weight ( $\beta=0.35, P=0.008), \mathrm{FFM}(\beta=0.38, P=0.004)$ and aerobic fitness as measured by absolute $\mathrm{VO}_{2 \text { peak }}(\beta=0.43, P<0.001)$ and maximal power during the exercise test $(\beta=0.43, P<0.001$; Supplemental Table 1). Similar to post-exercise, post-rest energy intake differed by sex $(P<0.001)$. Absolute $\mathrm{VO}_{2 \text { peak }}(\beta=0.53, P=0.006)$ and maximal power $(\beta=0.54, P=0.005)$ were only associated with ad libitum energy intake in men, while in women, only habitual exercise minutes per week was a significant predictor of ad libitum energy intake $(\beta=-0.37, P=0.048)$.

\subsection{Predictors of the difference between post-exercise and post-rest energy intake}

The difference in total energy intake between exercise and rest was inversely associated with aerobic fitness as measured by relative $(\beta=-0.31, P=0.020)$ and absolute $(\beta=-0.35, P=0.008) V_{2} O_{2 \text { peak. }}$. The difference between exercise and rest was also positively associated with fasting PYY concentrations ( $\beta=0.33, P=0.038$; Supplemental Table 2, Figure 1F). Notably, significant associations were driven by men, and they were not significant for women (Supplemental Table 2). In men, above a $\mathrm{VO}_{2 \text { peak }}$ cut point of $40.9 \mathrm{~mL} / \mathrm{kg} / \mathrm{min}(3.0 \mathrm{~L} / \mathrm{min})$, post-rest energy intake was greater than post-exercise energy intake, while below the cut point, post-exercise energy intake was greater than post-rest energy intake. For PYY in men, post-exercise energy intake was greater than post-rest energy intake above the cut point of $118.6 \mathrm{pg} / \mathrm{mL}$, while below the cut point, post-rest energy intake was greater than post-exercise energy intake.

\section{Discussion}

The present study aimed to identify predictors of post-exercise energy intake and compensation in healthy adults following a single 45-minute aerobic exercise bout. Our results show that individuals with lower habitual exercise and/or higher fasting concentrations of PYY eat more after an acute exercise bout, even after accounting for the energy expended during the exercise bout. Notably, these biological and behavioral characteristics differentially affected post-exercise energy intake in men and women; habitual exercise behavior was only predictive of post-exercise energy intake in women whereas fasting PYY concentrations were only a significant predictor of post-exercise intake in men. For habitual exercise in women, every 30 min/week increase was associated with a decrease in post-exercise energy intake and compensation of $\sim 20 \mathrm{kcal}$. In men, albeit not siginificant, the trend in the association between habitual exercise and post-exercise energy intake was similar to that in women. Generally, the association between greater habitual exercise and lower post-exercise energy compensation is in line with our hypothesis and previous research. It has been shown that energy balance is better regulated at higher levels of PA-related energy expenditure due to better satiety signaling and the fact that exercise-induced food rewards and cravings play a less important role. $6,7,25,26$ It is noteworthy that accelerometer-measured MVPA did not confirm these results, as MVPA was not a significant predictor of postexercise energy intake. Overall, our sample showed relatively high average MVPA levels ( $330 \mathrm{~min} /$ week) at baseline, with $89 \%$ of participants above the established weekly recommendations of $150 \mathrm{~min}$ of MVPA and $54 \%$ even above $300 \mathrm{~min} /$ week. $^{27}$ It can be speculated whether self-reported exercise behavior (prospectively recorded) included certain types of (even high-intensity) exercise such as strength training, cycling, or swimming that were not (accurately) captured by the hip-worn Actigraph devices, as demonstrated before, ${ }^{14-16}$ and whether this contributed to exercise behavior (min/week) being a better predictor of post-exercise energy intake and compensation than overall MVPA. The generally high MVPA level suggests that most participants would be in the regulated zone of Mayer's curve, in which energy expenditure and energy intake are in balance. 6,7 Nevertheless, despite the overall high MVPA levels, there was substantial variability in the post-exercise energy intake, demonstrating that the single exercise session evoked a greater compensatory energy intake response in some participants than others.

The associations between fasting concentrations of appetite-regulating hormones and post-exercise energy intake are striking. Particularly the sex differences with a strong predictive value of PYY and adiponectin in men, explaining $78 \%$ and $44 \%$ of the variance in post-exercise energy intake, respectively ( $72 \%$ and $48 \%$ for energy compensation) but no significant associations with post-exercise energy intake in women were unexpected. In men, every ten $\mathrm{pg} / \mathrm{mL}$ increase in fasting PYY concentrations and every one $\mathrm{ng} / \mathrm{mL}$ increase in fasting adiponectin concentrations was associated with an increase in post-exercise energy intake of $96 \mathrm{kcal}$ and $53 \mathrm{kcal}$, respectively (energy compensation: $94 \mathrm{kcal}$ and $56 \mathrm{kcal}$, respectively). We are not aware of previous findings of similar sex differences in the association between fasting appetite-regulating hormones and (post-exercise) energy intake. In our study,

Page $9 / 13$ 
PYY concentrations did not differ by sex at baseline, which is in line with the literature. ${ }^{28}$ In the fasted state, circulating concentrations of PYY are usually low with rapid increases upon nutrient ingestion. ${ }^{28,29}$ During aerobic exercise, PYY has been shown to increase, with the appetite-suppressing effects lasting up to 5 hours post-exercise. ${ }^{30}$ It has been reported that sex differences exist in the PYY response to moderate-intensity exercise (bike ergometer at $65 \% \mathrm{VO}_{2 \mathrm{max}}$, similar to our study), with greater increases and a greater subsequent post-exercise appetite suppression in men compared to women. ${ }^{31}$ Importantly, however, the exercise-induced increases in PYY and associated appetite suppression do not always translate into de-facto decreases in postexercise energy intake. In fact, the majority of exercise studies show no change in energy intake after acute bouts of exercise, ${ }^{30}$ and it has been found in a review that $19 \%$ of such studies even report increases in energy intake while $16 \%$ show a decrease ( $65 \%$ no change).$^{32}$ Nevertheless, the strong associations between greater fasting concentrations of PYY (and adiponectin) and greater post-exercise energy intake and compensation as shown in the present study are still somewhat unexpected. Further research is needed to confirm our findings and examine why fasting PYY may affect energy intake and compensation after exercise but not after a no-exercise rest condition; also of interest is why PYY concentrations affect post-exercise energy intake and compensation differently in men than women, despite similar fasting concentrations.

Contrary to previous findings showing a general association between appetitive traits, and particularly disinhibition or uncontrolled eating, with overeating in the presence of food, ${ }^{33-35}$ appetitive traits (cognitive restraint, uncontrolled eating, and emotional eating) were not significant predictors of energy intake. This is similar to recent findings in adolescents in whom the three trait measures as assessed via the TFEQ-R18v2 were also not associated with ad libitum post-exercise energy intake. ${ }^{36}$ Further, physiological processes may override these appetitive traits in the post-exercise state; however, in our study, cognitive restraint, uncontrolled eating, or emotional eating were also not associated with ad libitum energy intake following the control condition involving rest.

Energy intake after rest was associated with weight and FFM as well as cardiorespiratory fitness $\left(\mathrm{VO}_{2 \text { peak }}\right.$ and maximal power). Of note, only absolute but not relative $\mathrm{VO}_{2 \text { peak }}$ were significant predictors of post-rest energy intake, suggesting that this association was driven by the significant predictors of weight, FFM, and sex, which have repeatedly been shown to predict meal size and single meal food intake. ${ }^{22-24}$ When assessing predictors of the difference in energy intake between after exercise and after rest, we found significant associations with cardiorespiratory fitness, in this case, both relative and absolute $\mathrm{VO}_{2 \text { peak, }}$, for men but not women. Further, the associations revealed a turning point at $40.9 \mathrm{~mL} / \mathrm{kg} / \mathrm{min}$ or $3.0 \mathrm{~L} / \mathrm{min}$, respectively. Individuals with average-to-above-average fitness seem to have reduced total energy intake post-exercise compared to post-rest while those with below-average fitness seem to have increased total energy intake post-exercise compared to post-rest. The finding of greater post-exercise energy intake in individuals with lower fitness compared to those with higher fitness is supported by previous findings showing that individuals with higher compared to lower fitness (mean $\mathrm{VO}_{2 \text { peak }}$ of 51.6 vs. 37.0) compensated after an acute exercise session compared to after rest. ${ }^{37}$ Additionally, this relationship may be influenced by higher body fat accumulation, which often co-occurs with lower fitness and has been shown to predict greater post-exercise energy compensation due to a progressively increasing impairment of energy balance. ${ }^{38}$

Overall, our results may help identify individuals who are likely to show post-exercise energy compensation and help explain why this adverse response to exercise occurs in some individuals but not others. To prevent increased post-exercise energy intake, countermeasures such as the selection and preparation of the post-exercise meal ahead of the exercise session may be beneficial, as demonstrated previously. ${ }^{13,39}$ Strengths of the present study include the crossover design, the relatively balanced sample of men and women, and the comprehensive analysis of the contribution of anthropometric, behavioral, cognitive, and endocrine factors at baseline to post-exercise energy intake. Limitations include the relatively small sample, particularly for the appetite-regulating hormone data, and the relative lack of racial and age-related diversity. However, we specifically chose to recruit a convenience sample of young adults to minimize the impact of age, as it has been shown that exercise-induced consequences of hunger, satiety, and compensation differentially affect adults aged $60+$ years. ${ }^{40}$

Nevertheless, future studies with larger and more diverse samples should examine potential differences in predictors of post-exercise energy intake by race/ethnicity and age. Further, it would be interesting to assess how different exercise modalities (type, intensity, duration) with similar energy expenditure affect post-exercise food intake and compensation and whether the found associations hold true for repeated exercise bouts.

\section{Conclusions}

Biological and behavioral characteristics differentially affect post-exercise energy intake in men and women. In women, habitual exercise behavior predicts post-exercise energy intake, with more exercise protecting against compensatory eating. In men, appetite-regulating hormones, and specifically PYY and adiponectin, play a role in the energy intake response to acute exercise, even when measured before exercise and after controlling for post-rest energy intake. Our results can help identify individuals who are more likely to (over-) compensate for the energy expended in exercise via increased post-exercise energy intake allowing to deploy targeted countermeasures ahead of time.

\section{Declarations Conflicts of Interest}

The authors have no conflicts of interest to declare.

\section{Financial support}


This research was funded by the University of Nebraska Food for Health Collaboration Initiative.The funding source had no role in the design and conduct of the study; collection, management, analysis, and interpretation of the data; or preparation, review, or approval of the manuscript.

\section{Ethical statement}

The study was approved by the University of Nebraska-Lincoln's Institutional Review Board (project number 17239) and written informed consent was obtained from all participants before participation in the study. All study procedures were conducted in accordance with the Declaration of Helsinki.

\section{Author contributions}

K.K., J.R.S., J.B.B., and J.A.F. acquired funding. K.K. and J.R.S. designed the study and S.E.B., J.A.F., J.B.B., and K.K. collected data. C.H. conducted statistical analyses, drafted the manuscript, and created tables and figures. K.K., S.E.B., J.A.F., J.R.S., and J.B.B. provided critical revision of the manuscript for important intellectual content. All authors have read and agreed to the published version of the manuscript.

\section{Data availability}

The data that support the findings of this study are available from the corresponding author on reasonable request

\section{References}

1. Dorling J, Broom DR, Burns SF, Clayton DJ, Deighton K, James LJ, King JA, Miyashita M, Thackray AE, Batterham RL, Stensel DJ. Acute and Chronic Effects of Exercise on Appetite, Energy Intake, and Appetite-Related Hormones: The Modulating Effect of Adiposity, Sex, and Habitual Physical Activity. Nutrients. 2018;10:1140.

2. King JA, Deighton K, Broom DR, Wasse LK, Douglas JA, Burns SF, Cordery PA, Petherick ES, Batterham RL, Goltz FR, Thackray AE, Yates T, Stensel DJ. Individual Variation in Hunger, Energy Intake, and Ghrelin Responses to Acute Exercise. Med Sci Sports Exerc. 2017;49:1219-1228.

3. Church TS, Martin CK, Thompson AM, Earnest CP, Mikus CR, Blair SN. Changes in Weight, Waist Circumference and Compensatory Responses with Different Doses of Exercise among Sedentary, Overweight Postmenopausal Women. PLoS ONE. 2009;4:e4515.

4. Martin CK, Johnson WD, Myers CA, Apolzan JW, Earnest CP, Thomas DM, Rood JC, Johannsen NM, Tudor-Locke C, Harris M, Hsia DS, Church TS. Effect of different doses of supervised exercise on food intake, metabolism, and non-exercise physical activity: The E-MECHANIC randomized controlled trial. Am J Clin Nutr. 2019;110:583-592.

5. Edholm OG. Energy expenditure in relation to nutrition. Proc Nutr Soc. 1956;15:80-83.

6. Mayer J, Roy P, Mitra KP. Relation between caloric intake, body weight, and physical work: studies in an industrial male population in West Bengal. Am J Clin Nutr. 1956;4:169-175.

7. Church T, Martin CK. The Obesity Epidemic: A Consequence of Reduced Energy Expenditure and the Uncoupling of Energy Intake? Obes Silver Spring Md. 2018;26:14-16.

8. French SA, Epstein LH, Jeffery RW, Blundell JE, Wardle J. Eating Behavior Dimensions: Associations With Energy Intake And Body Weight: A Review. Appetite. 2012;59:541-549.

9. Feig EH, Piers AD, Kral TVE, Lowe MR. Eating in the absence of hunger is related to loss-of-control eating, hedonic hunger, and short-term weight gain in normal-weight women. Appetite. 2018;123:317-324.

10. Schubert MM, Sabapathy S, Leveritt M, Desbrow B. Acute exercise and hormones related to appetite regulation: a meta-analysis. Sports Med Auckl NZ. 2014;44:387-403.

11. Douglas JA, King JA, Clayton DJ, Jackson AP, Sargeant JA, Thackray AE, Davies MJ, Stensel DJ. Acute effects of exercise on appetite, ad libitum energy intake and appetite-regulatory hormones in lean and overweight/obese men and women. Int J Obes 2005. 2017;41:1737-1744.

12. Deighton K, Stensel DJ. Creating an acute energy deficit without stimulating compensatory increases in appetite: is there an optimal exercise protocol? Proc Nutr Soc. 2014;73:352-358.

13. Koehler K, Beckford SE, Thayer E, Martin AR, Boron JB, Stevens JR. Exercise Shifts Hypothetical Food Choices toward Greater Amounts and More Immediate Consumption. Nutrients. 2021;13:347.

14. Berntsen S, Hageberg R, Aandstad A, Mowinckel P, Anderssen SA, Carlsen K-H, Andersen LB. Validity of physical activity monitors in adults participating in free-living activities. Br J Sports Med. 2010;44:657-664.

15. Herman Hansen B, Børtnes I, Hildebrand M, Holme I, Kolle E, Anderssen SA. Validity of the ActiGraph GT1M during walking and cycling. J Sports Sci. 2014;32:510-516.

16. Höchsmann C, Knaier R, Infanger D, Schmidt-Trucksäss A. Validity of smartphones and activity trackers to measure steps in a free-living setting over three consecutive days. Physiol Meas. 2020;41:015001.

17. Achten J, Jeukendrup AE. Maximal fat oxidation during exercise in trained men. Int J Sports Med. 2003;24:603-608.

18. Borg GA. Psychophysical bases of perceived exertion. Med Sci Sports Exerc. 1982;14:377-381.

Page $11 / 13$ 
19. Stunkard AJ, Messick S. The three-factor eating questionnaire to measure dietary restraint, disinhibition and hunger. J Psychosom Res. 1985;29:7183.

20. Karlsson J, Persson LO, Sjöström L, Sullivan M. Psychometric properties and factor structure of the Three-Factor Eating Questionnaire (TFEQ) in obese men and women. Results from the Swedish Obese Subjects (SOS) study. Int J Obes Relat Metab Disord J Int Assoc Study Obes. 2000;24:1715-1725.

21. Cappelleri JC, Bushmakin AG, Gerber RA, Leidy NK, Sexton CC, Lowe MR, Karlsson J. Psychometric analysis of the Three-Factor Eating QuestionnaireR21: results from a large diverse sample of obese and non-obese participants. Int J Obes 2005. 2009;33:611-620.

22. Weise CM, Hohenadel MG, Krakoff J, Votruba SB. Body composition and energy expenditure predict ad-libitum food and macronutrient intake in humans. Int J Obes 2005. 2014;38:243-251.

23. Blundell JE, Caudwell P, Gibbons C, Hopkins M, Näslund E, King NA, Finlayson G. Body composition and appetite: fat-free mass (but not fat mass or $\mathrm{BMI}$ ) is positively associated with self-determined meal size and daily energy intake in humans. Br J Nutr. 2012;107:445-449.

24. Hopkins M, Finlayson G, Duarte C, Whybrow S, Ritz P, Horgan GW, Blundell JE, Stubbs RJ. Modelling the associations between fat-free mass, resting metabolic rate and energy intake in the context of total energy balance. Int J Obes. 2016;40:312-318.

25. Beaulieu K, Hopkins M, Long C, Blundell J, Finlayson G. High Habitual Physical Activity Improves Acute Energy Compensation in Nonobese Adults. Med Sci Sports Exerc. 2017;49:2268-2275.

26. Beaulieu K, Oustric P, Finlayson G. The Impact of Physical Activity on Food Reward: Review and Conceptual Synthesis of Evidence from Observational, Acute, and Chronic Exercise Training Studies. Curr Obes Rep. 2020;9:63-80.

27. Bull FC, Al-Ansari SS, Biddle S, Borodulin K, Buman MP, Cardon G, Carty C, Chaput J-P, Chastin S, Chou R, Dempsey PC, DiPietro L, Ekelund U, Firth J, Friedenreich CM, Garcia L, Gichu M, Jago R, Katzmarzyk PT, Lambert E, Leitzmann M, Milton K, Ortega FB, Ranasinghe C, Stamatakis E, Tiedemann A, Troiano RP, Ploeg HP van der, Wari V, Willumsen JF. World Health Organization 2020 guidelines on physical activity and sedentary behaviour. Br J Sports Med. 2020;54:1451-1462.

28. Cooper JA. Factors affecting circulating levels of peptide YY in humans: a comprehensive review. Nutr Res Rev. 2014;27:186-197.

29. Druce MR, Small CJ, Bloom SR. Minireview: Gut peptides regulating satiety. Endocrinology. 2004;145:2660-2665.

30. Stensel D. Exercise, Appetite and Appetite-Regulating Hormones: Implications for Food Intake and Weight Control. Ann Nutr Metab. 2010;57:36-42.

31. Hazell TJ, Townsend LK, Hallworth JR, Doan J, Copeland JL. Sex differences in the response of total PYY and GLP-1 to moderate-intensity continuous and sprint interval cycling exercise. Eur J Appl Physiol. 2017;117:431-440.

32. Blundell JE, King NA. Physical activity and regulation of food intake: current evidence. Med Sci Sports Exerc. 1999;31:S573.

33. Vainik U, García-García I, Dagher A. Uncontrolled eating: a unifying heritable trait linked with obesity, overeating, personality and the brain. Eur J Neurosci. 2019;50:2430-2445.

34. Garcia-Garcia I, Neseliler S, Morys F, Dadar M, Yau YHC, Scala SG, Zeighami Y, Sun N, Collins DL, Vainik U, Dagher A. Relationship between impulsivity, uncontrolled eating and body mass index: a hierarchical model. Int J Obes 2005. 2022;46:129-136.

35. Brunner EJ, Maruyama K, Shipley M, Cable N, Iso H, Hiyoshi A, Stallone D, Kumari M, Tabak A, Singh-Manoux A, Wilson J, Langenberg C, Wareham N, Boniface D, Hingorani A, Kivimäki M, Llewellyn C. Appetite disinhibition rather than hunger explains genetic effects on adult BMI trajectory. Int $\mathrm{J}$ Obes 2005. 2021;45:758-765.

36. Fearnbach N, Staiano AE, Johannsen NM, Hsia DS, Beyl RA, Carmichael OT, Martin CK. Predictors of Post-Exercise Energy Intake in Adolescents Ranging in Weight Status from Overweight to Severe Obesity. Nutrients. 2022;14:223.

37. Charlot K, Chapelot D. Energy compensation after an aerobic exercise session in high-fat/low-fit and low-fat/high-fit young male subjects. Br J Nutr. 2013;110:1133-1142.

38. Careau V, Halsey LG, Pontzer H, Ainslie PN, Andersen LF, Anderson LJ, Arab L, Baddou I, Bedu-Addo K, Blaak EE, Blanc S, Bonomi AG, Bouten CVC, Buchowski MS, Butte NF, Camps SGJA, Close GL, Cooper JA, Das SK, Cooper R, Dugas LR, Eaton SD, Ekelund U, Entringer S, Forrester T, Fudge BW, Goris AH, Gurven M, Hambly C, El Hamdouchi A, Hoos MB, Hu S, Joonas N, Joosen AM, Katzmarzyk P, Kempen KP, Kimura M, Kraus WE, Kushner RF, Lambert EV, Leonard WR, Lessan N, Martin CK, Medin AC, Meijer EP, Morehen JC, Morton JP, Neuhouser ML, Nicklas TA, Ojiambo RM, Pietiläinen KH, Pitsiladis YP, Plange-Rhule J, Plasqui G, Prentice RL, Rabinovich RA, Racette SB, Raichlen DA, Ravussin E, Reilly JJ, Reynolds RM, Roberts SB, Schuit AJ, Sjödin AM, Stice E, Urlacher SS, Valenti G, Van Etten LM, Van Mil EA, Wells JCK, Wilson G, Wood BM, Yanovski J, Yoshida T, Zhang X, Murphy-Alford AJ, Loechl CU, Luke AH, Rood J, Sagayama H, Schoeller DA, Wong WW, Yamada Y, Speakman JR. Energy compensation and adiposity in humans. Curr Biol. 2021;31:4659-4666.e2.

39. Gustafson CR, Rakhmatullaeva N, Beckford SE, Ammachathram A, Cristobal A, Koehler K. Exercise and the Timing of Snack Choice: Healthy Snack Choice is Reduced in the Post-Exercise State. Nutrients. 2018;10:E1941.

40. Hubner S, Boron JB, Koehler K. The Effects of Exercise on Appetite in Older Adults: A Systematic Review and Meta-Analysis. Front Nutr. $2021 ; 8: 734267$.

\section{Figures}


Post-Exercise Energy Intake
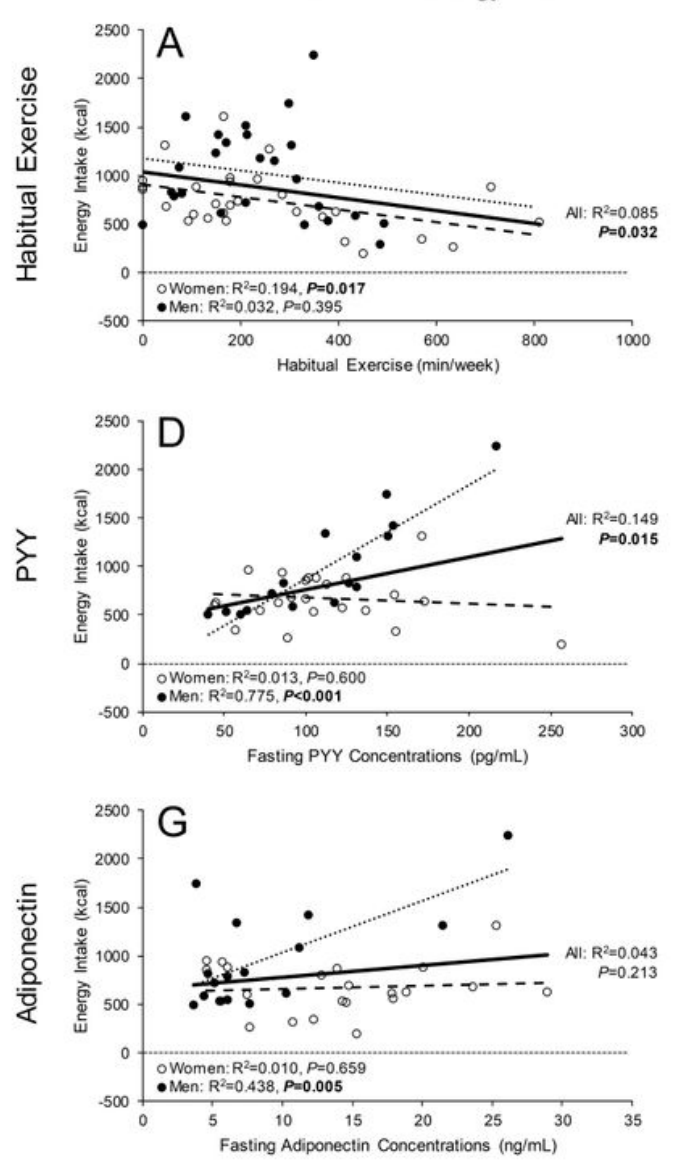

Compensatory Energy Intake
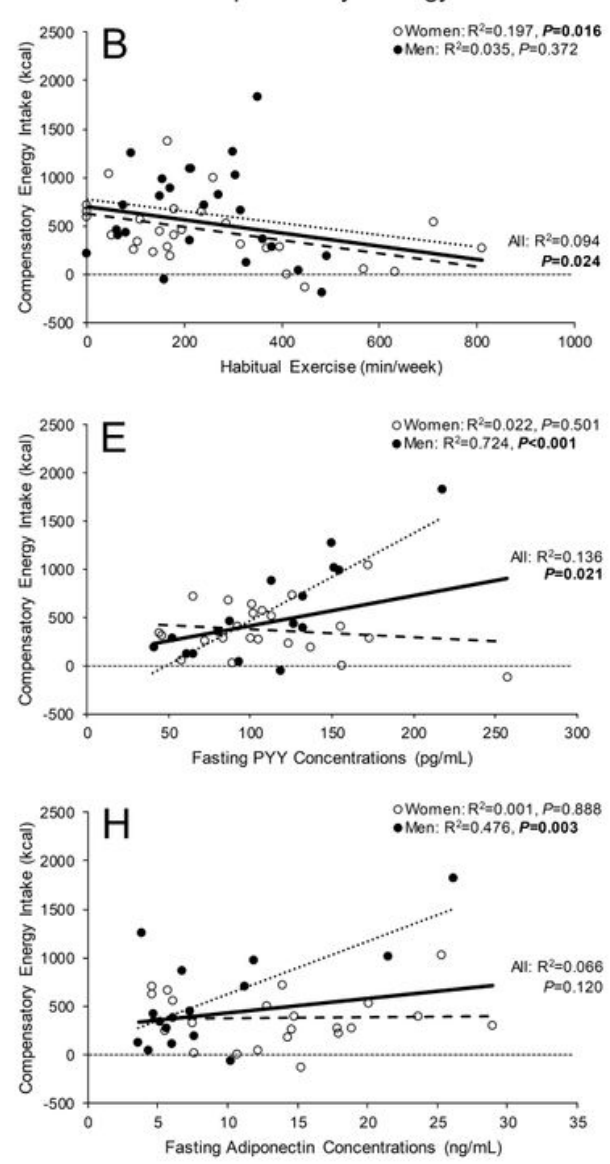

Post-Exercise - Post-Rest Energy Intake
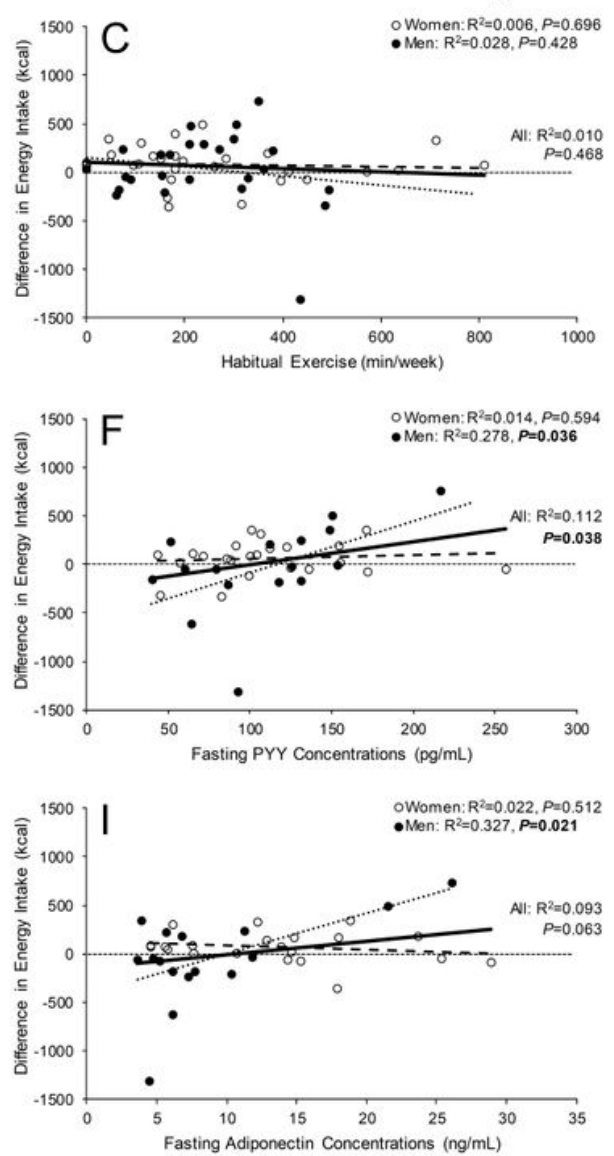

\section{Figure 1}

Associations between habitual exercise, fasting PYY concentrations, and fasting adiponectin concentrations and post-exercise energy intake, compensatory energy intake (post-exercise energy intake - exercise energy expenditure), and the difference between post-exercise and post-rest energy intake. Regression lines are displayed for the entire sample (solid line), for men (dotted line), and for women (dashed line).

\section{Supplementary Files}

This is a list of supplementary files associated with this preprint. Click to download.

- SupplementalTable1.docx

- SupplementalTable2.docx 